\title{
The Effect of the Drug Estrofan on the Content of Nitric Oxide (II), Sex Hormones, Biochemical Parameters and Their Relationship in Cows
}

\author{
I.T. Sibgatullin*, R.Ya. Gilmutdinov and T.M. Zakirov
}

Kazan State Academy of Veterinary Medicine named after N.E. Bauman, 35, Siberian tract, Kazan, 420029, RT, Russia

\begin{abstract}
The physiological functions of nitric oxide (II) are diverse, and its therapeutic uses continue to expand. Many methods have been found to regulate the production of this compound, both physiological, regulated by the body itself, and as a result of various, drug and non-drug, external influences, correcting the ability of cells, organs and tissues to produce nitric oxide (II). The drug estrophan (synthetic prostaglandin PGF2 $\alpha$ ) has an activating effect on the nitric oxide (II) system and on sex hormones that regulate the reproductive functions of cows. The introduction of estrophan to cows is accompanied by an increase in the synthesis of nitric oxide (II), estrogen in the body of cows, and a decrease in progesterone. A high level of interrelation of estrogen with nitric oxide (II) was shown, the value of the correlation coefficient is $(r=0.552 ; p<0.05)$. Correlation of the level of nitric oxide (II) with estrogen allows making an assumption of its bioregulatory functions, including the sexual cycle. The introduction of estrophan had no effect on the biochemical parameters in the blood serum of cows.
\end{abstract}

\section{Introduction}

The problems of reproduction and prevention of infertility in cows under the conditions of modern industrial maintenance and exploitation, even with the significant achievements in the field of reproductive physiology, do not lose their relevance for animal husbandry [4]. More than half of the forcedly slaughtered individuals have various gynecological disorders. One of the most common manifestations of reproductive dysfunction is low fertility and high embryonic mortality $[5,6,9]$. With a significant number of scientific works devoted to the influence of various biologically active substances on the reproductive function of dairy cows, there is still no consensus on both the mechanisms of action and the effectiveness of these drugs $[1,2,14,15]$. In this regard, in addition to creating favorable conditions for the implementation of their own reproductive capabilities of animals, it is promising to adjust biotechnological methods for regulating reproductive function and search for the most effective, environmentally friendly methods of preventing and treating the corresponding diseases.

Nitric oxide (II) is an important biological mediator involved in many physiological processes. This secondary messenger, unique in its nature and mechanisms of action, is involved in the implementation of many important physiological functions of the body, such as vasodilation, neurotransmission, decreased platelet aggregation, immune system reactivity, smooth muscle tone regulation, memory state, etc. A major scientific achievement was the discovery of universal bioregulatory properties. its endogenous variant, which opens up the possibility of an innovative approach in the treatment of certain diseases in humans and animals [5]. At present, female sex is considered as an independent factor in the protection and resistance of animals to stress, as well as diseases of various etiologies. This phenomenon is associated, among other things, with the activity of the system for generating nitric oxide (II) [9-13].

The relationship between prostaglandins involved in a large number of reproductive processes and vasoactive substances, including nitric oxide (II), is known. The reaction of cows to exogenous prostaglandins PGF $2 \alpha$ is manifested by the normalization of the functional activity of the adrenal glands, activation of lipid peroxidation processes, generation of nitric oxide (II), and increased glucose utilization. Regarding reproductive functions, administration of PGF2 $\alpha$ preparations to cows after childbirth can reduce postpartum complications, increase fertility, including a decrease in the number of remaining infertile animals $[6,11,12,16]$.

In the presented work, the effect of prostaglandin PGF2 $\alpha$ (estrophan drug) on the content of nitric oxide (II), sex hormones and their relationship in cows was studied.

\section{Materials and methods}

The research was carried out on 30 black-and-white cows at the age of $4-8$ years at the farm "Khadiev D.M."

Corresponding author: sib.ilnaz@yandex.ru 
in Kukmorsky district of the Republic of Tatarstan in the winter stall period in 2019. The animals were kept in standard rooms on a leash, with the provision of walks in the corral.

In the experiment, the content of nitric oxide (II) and the hormonal status of cows under the influence of PGF2 $\alpha$ (estrophan drug), which was injected intramuscularly at a dose of $2 \mathrm{ml}$, were determined. The drug promotes the resorption of the corpus luteum and induces the onset of estrum and ovulation (arousal stage) after 48-60 hours. It is excreted from the body after 24 hours. Before the experiment and every 6, 12 and 24 hours after its administration, the animals were taken venous blood from the jugular vein. During the experiments, physiological, biochemical, and spectrophotometric methods were used.

The total concentration of nitrate and nitrite anions in blood serum was determined by reducing nitrates to nitrites with zinc dust manufactured by Arsenal LLC, (Ukraine). For this, $1 \mathrm{ml}$ of $6 \%$ zinc sulfate solution of Spectr-Khim LLC (Russia) was added to $1 \mathrm{ml}$ of plasma and left for 1 hour at a temperature below $15^{\circ} \mathrm{C}$ and then centrifuged at $6000 \mathrm{rpm}$ (3000 g). An equivalent amount of sodium hydroxide ( $300 \mu \mathrm{L}$ of $1 \mathrm{~N}$ solution) was added to the supernatant, centrifuged, and $1 \mathrm{~mL}$ of the sedimentary liquid was transferred into a plastic tube corresponding to the numbering with $0.11 \mathrm{~g}$ of zinc dust, $0.5 \mathrm{~mL}$ of ammonia buffer, and $20 \mu \mathrm{L}$ of an ammonia complex of copper sulfate previously added thereto. The tubes were sealed and shaken for 30 minutes. Zinc dust was precipitated by centrifugation at $3000 \mathrm{rpm}$ for 10 min. Then, $1 \mathrm{ml}$ of sodium acetate solution and $1 \mathrm{ml}$ of 1-naphthylamine hydrochloric acid solution from Vekton LLC (Russia) were added to each tube. The optical density was measured after $30 \mathrm{~min}$ on a Photoelectric photometer KFK-3-01-ZOMZ (JSC “ZOMZ”, Russia) at a wavelength of $520 \mathrm{~nm}$.

In the blood serum, the content of progesterone, estrogen and biochemical parameters was determined by spectrometry on a biochemical analyzer "Bi-An" ABkhFk-02-NPP-TM (CJSC NPP "Technomedica", Russia) with a set of reagents by OOO "Olvex Diagnosticum" (Russia).

The concentration of total protein in the blood serum was determined using a biuret reaction with a set of reagents by "Olvex" (Russia), followed by measuring the optical density on a biochemical analyzer "Bi-An" (Russia) at a wavelength of $540 \mathrm{~nm}$.

The concentration of urea in the blood serum was determined by the enzymatic kinetic method with a set of reagents by "Olvex" (Russia). Urea was hydrolyzed in the presence of urease to form ammonium ions and $\mathrm{CO}_{2}$. Ammonium ions react with $\alpha$-ketoglutarate and $\mathrm{NADH}$ in the presence of glutamate dehydrogenase (GLDH) to form glutamate and NAD. The optical density of the samples and the standard was measured after 30 seconds and after 60 seconds on a biochemical analyzer "Bi-An" (Russia) at a wavelength of $340 \mathrm{~nm}$.

The concentration of creatinine in the blood serum was determined by the kinetic method with a set of reagents by "Olvex" (Russia). In an alkaline medium, creatinine interacts with picric acid to form a red-colored product, the optical density of which was measured after 30 seconds and 90 seconds on a Bi-An biochemical analyzer (Russia) at a wavelength of $510 \mathrm{~nm}$. The difference in optical density was used to calculate the concentration of creatinine in the sample and in the standard.

The AST concentration in the blood serum was determined using the Olvex reagent kit (Russia). Under the action of the AST enzyme, as a result of transamination, the amino group is transferred from aspartate to $\alpha$-ketoglutarate. The oxaloacetate formed in this reaction is converted into malate with the participation of the enzyme malate dehydrogenase and the coenzyme $\mathrm{NADH}_{2}$. The oxidation rate of $\mathrm{NADH}_{2}$ in the course of the second reaction is determined by the decrease in the optical density of the reaction medium at $340 \mathrm{~nm}$ and is proportional to the activity of AST.

The concentration of ALT in the blood serum was determined with a set of reagents "Olvex" (Russia). Under the action of the enzyme ALT, as a result of transamination, the amino group is transferred from alanine to $\alpha$-ketoglutarate. The pyruvate formed in this reaction is converted into lactate with the participation of the enzyme LDH and the coenzyme $\mathrm{NADH}_{2}$. The oxidation rate of $\mathrm{NADH}_{2}$ in the course of the second reaction is determined by the decrease in the optical density of the reaction medium at $340 \mathrm{~nm}$ and is proportional to the ALT activity.

The concentration of calcium in the blood serum was determined by a unified colorimetric method with a set of reagents "Olvex" (Russia). Calcium in an alkaline medium forms a colored complex with o-cresolphthalein complexone. The intensity of color by optical density at $570 \mathrm{~nm}$ on a biochemical analyzer "Bi-An" (Russia) is proportional to the concentration of calcium in the sample.

The concentration of phosphorus in the blood serum was determined by the spectrophotometric method on the Biochemical Photometric Kinetic Analyzer Bi-An (Russia) with the Olvex reagent kit (Russia). The method is based on the ability of phosphate ions to form a phosphoromolybdenum complex in an acidic medium with ammonium molybdate in the presence of a detergent, the optical density of which at a wavelength of $340 \mathrm{~nm}$ is proportional to the concentration of inorganic phosphorus in the sample under study.

Biometric processing of the experimental results was carried out using the Student's t test; the differences were considered significant at the $\mathrm{p}<0.05$ level. To identify the relationship between the indicators, the method of correlation analysis was used. The correlation coefficient was determined by the Pearson square method using the MS Excel program, and three ranges of the communication level were identified: strong - from \pm 0.7 to \pm 1.0 ; medium - from \pm 0.3 to \pm 0.699 and weak from 0 to \pm 0.299 .

\section{Results}

Earlier, we found that the content of nitric oxide (II) in the blood of cows depends on the stage of the sexual cycle. The maximum activity of nitric oxide (II) noted in 
the stage of excitation is $75.5 \pm 5.36 \mu \mathrm{mol} / \mathrm{L}(\mathrm{p}<0.05)$, in the stage of inhibition is $73.5 \pm 2.35 \mu \mathrm{mol} / \mathrm{L}$ $(\mathrm{p}<0.05)$, and in the stage of equilibration is $70.0 \pm 6.66 \mu \mathrm{mol} / \mathrm{L}(\mathrm{p}>0.05)$.

Since prostaglandins are a group of acidic lipids that are involved in a large number of reproductive processes, the next stage we studied the state of the nitroxydergic system under the action of estrophan, since the understanding of the mechanism is not yet perfect, in addition, the relationship between prostaglandins and other vasoactive substances, such as nitric oxide, complicates this area and is not fully understood.

The results of a study of the effect of estrophan, which stimulates estrum in animals, on the content of sex hormones in the blood serum of cows are presented in Table 1 .

Table 1.The amount of estrogen and progesterone in the serum of cows after administration of the drug estrofan

\begin{tabular}{|c|c|c|}
\hline Study period & $\begin{array}{c}\text { Progesterone } \\
{[\mathbf{n g} / \mathbf{m l}]}\end{array}$ & $\begin{array}{c}\text { Estrogen } \\
{[\mathbf{p g} / \mathbf{m l}]}\end{array}$ \\
\hline $\begin{array}{c}\text { Before } \\
\text { administration }\end{array}$ & $3.6 \pm 0.83$ & $29.4 \pm 1.01$ \\
\hline After 6 hours & $3.1 \pm 1.27$ & $35.8 \pm 3.42^{*}$ \\
\hline After 12 hours & $2.5 \pm 1.49$ & $39.3 \pm 2.73^{*}$ \\
\hline After 24 hours & $2.1 \pm 1.37^{*}$ & $41.4 \pm 1.17^{*}$ \\
\hline
\end{tabular}

Note: $*-p<0.05$ compared to cows before drug administration

The reaction of the endocrine system of cows to the introduction of prostaglandin PGF $2 \alpha$ was characterized by a pronounced increase in the content of estrogen in the blood serum and a decrease in the concentration of progesterone. The amount of progesterone in the blood serum of cows tended to decrease after the introduction of estrophan by 1.2 times $(p>0.05)$ after 6 hours, by 1.4 times $(\mathrm{p}>0.05)$ after 12 hours and by 1.7 times $(\mathrm{p}<0.05) 24$ hours after exposure, compared to values before administration, which is associated with accelerated lysis of the corpus luteum during this time. In turn, a decrease in the content of progesterone affects the pituitary gland, leading to an earlier release of gonadotropic hormones, and, as a result, an accelerated maturation of follicles, which was manifested by their increased secretion of estrogen. The dynamics of changes in estrogen content was characterized by an increase in concentration by 1.2 times $(p<0.05) 6$ hours after drug administration; by 1.3 times $(\mathrm{p}<0.05)$ after 12 hours, by $1.4 \quad(\mathrm{p}<0.05)$ times after 24 hours, compared to the values before administration. The results of studying the effect of estrophan on the content of nitric oxide (II) are presented in Table 2.

Table 2. The amount of nitric oxide (II) in the blood serum of cows after the administration of the drug estrophan

\begin{tabular}{|c|c|}
\hline Study period & Nitric oxide (II) $[\boldsymbol{\mu m o l} / \mathbf{I}]$ \\
\hline Before administration & $71.6 \pm 1.63$ \\
\hline After 6 hours & $79.3 \pm 3.74^{*}$ \\
\hline After 12 hours & $82.4 \pm 2.23^{*}$ \\
\hline After 24 hours & $89.6 \pm 3.74^{*}$ \\
\hline
\end{tabular}

Note: $*-p<0.05$ compared to cows before drug

administration
According to the data in Table 2, it follows that the administration of estrophan is accompanied by the activation of the synthesis of nitric oxide (II) in the body of cows. It was found that the concentration of this compound increased by a factor of $1.1(\mathrm{p}<0.05)$ after 6 hours after the drug was administered; by 1.2 times $(p<0.05)$ after 12 hours, and after 24 hours this indicator increased by 1.3 times ( $p<0.05)$, as compared to the values before the introduction. It can be assumed that, against the background of a decrease in the concentration of estrophan in blood serum, the content of nitric oxide (II) also decreases $(\mathrm{p}<0.05)$.

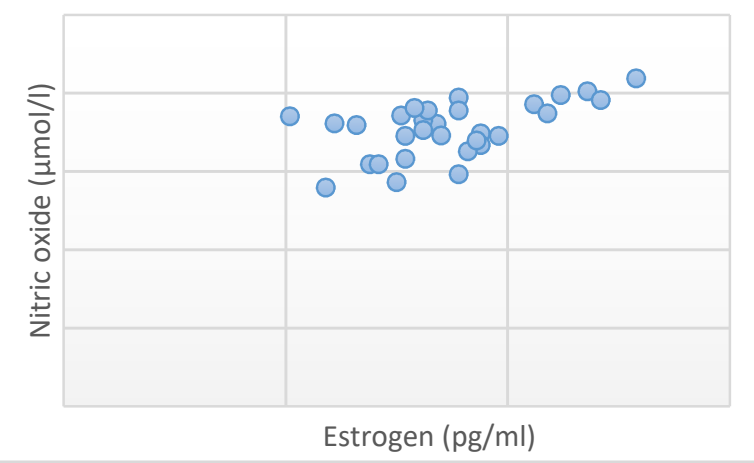

Fig. 1. Scattering of nitric oxide (II) and estrogen

Figure 1 shows the correlation in the form of a scatter plot with a high correlation between estrogen and nitric oxide (II) and the correlation coefficient value of $\mathrm{r}=$ 0.552 ( $\mathrm{p}<0.05)$. Probably, taking into account the literature data [5-7], estrogen has a powerful potentiating effect on the production of nitric oxide (II), the nature of changes in the activity of which is determined by the needs of organs for blood supply: as a rule, a high level of production of nitric oxide (II) corresponds to the period when there is a high functional activity of the corresponding organ.

Figure 2 shows the correlation in the form of a scatter plot. There is also a significant, but negative, correlation between the level of progesterone and nitric oxide (II) $(\mathrm{r}=-0.521 ; \mathrm{p}<0.05)$. It is possible that the high activity of nitric oxide (II) at the systemic level reduces or prevents the luteolytic effect of prostaglandins and thus maintains an adequate level of progesterone, but the exact mechanisms by which it exerts this effect remains to be studied in detail.

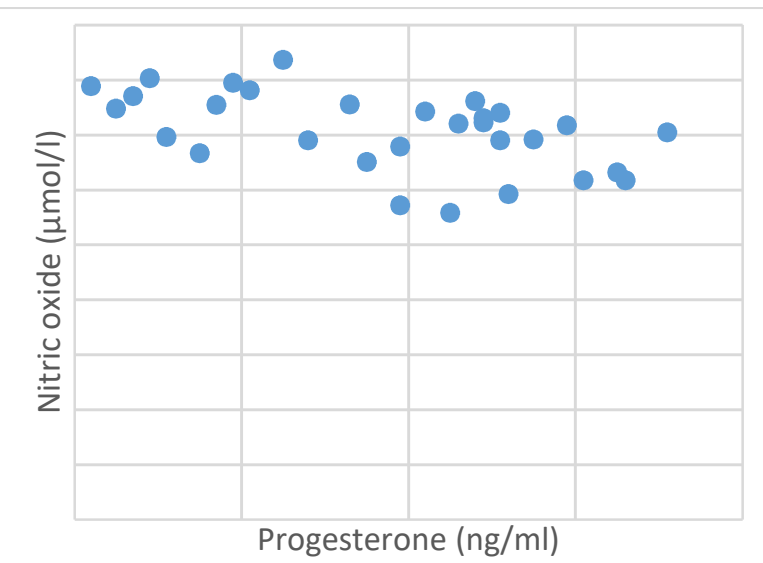

Fig. 2. Scatter plot of nitric oxide (II) and progesterone 
The results of studying the effect of estrophan on the concentration of biochemical parameters in the blood serum of cows are presented in Table 3.

Table 3. Concentration of biochemical parameters in the blood serum of cows after administration of the drug estrophan

\begin{tabular}{|c|c|c|c|c|}
\hline Indicators & Before administration & $\begin{array}{c}\text { After } \\
\text { 6 hours }\end{array}$ & $\begin{array}{c}\text { After } \\
\mathbf{1 2} \text { hours }\end{array}$ & $\begin{array}{c}\text { After } \\
\mathbf{2 4} \text { hours }\end{array}$ \\
\hline Total protein, g/l & $63.31 \pm 1.20$ & $66.31 \pm 1.54$ & $66.67 \pm 1.66$ & $73.85 \pm 1.67$ \\
\hline Albumin, g/l & $38.62 \pm 1.33$ & $39.68 \pm 0.48$ & $38.47 \pm 0.42$ & $41.73 \pm 0.56$ \\
\hline Globulins, g/l & $24.68 \pm 2.19$ & $26.62 \pm 0.33$ & $28.20 \pm 0.47$ & $32.12 \pm 0.44$ \\
\hline Urea, mmol/l & $3.22 \pm 0.34$ & $3.08 \pm 0.19$ & $2.93 \pm 0.16$ & $2.88 \pm 0.24$ \\
\hline Creatinine, mmol/l & $83.43 \pm 2.93$ & $84.5 \pm 3.96$ & $89.63 \pm 4.93$ & $92.43 \pm 4.12$ \\
\hline Glucose, mmol/l & $2.13 \pm 0.06$ & $1.82 \pm 0.17$ & $2.33 \pm 0.12$ & $2.13 \pm 0.24$ \\
\hline ALT, IU/l & $30.34 \pm 1.76$ & $34.33 \pm 2.08$ & $32.23 \pm 2.52$ & $33.54 \pm 2.09$ \\
\hline AST, IU/l & $67.86 \pm 3.72$ & $63.24 \pm 3.24$ & $69.67 \pm 2.84$ & $62.65 \pm 4.62$ \\
\hline ALP, IU/l & $71.74 \pm 7.88$ & $65.17 \pm 3.06$ & $66.67 \pm 4.71$ & $67.23 \pm 6.12$ \\
\hline Calcium, mmol/l & $2.45 \pm 0.07$ & $2.98 \pm 0.05$ & $3.03 \pm 0.09$ & $3.15 \pm 0.06$ \\
\hline Phosphorus, mmol/l & $2.03 \pm 0.05$ & $1.74 \pm 0.11$ & $1.78 \pm 0.13$ & $1.51 \pm 0.09$ \\
\hline
\end{tabular}

All biochemical parameters in the blood serum of cows after the injection of prostaglandin PGF2 $\alpha$ were within the physiological norm. The amount of total protein in the blood serum of cows tended to increase after the introduction of estrophan after 24 hours by 1.2 times ( $p>0.05)$. Also, in the blood of the cows of the experimental group, there was a slight tendency to an increase in the concentration of creatinine and a decrease in urea (Table 3).

\section{Conclusion}

The administration of prostaglandin PGF $2 \alpha$ preparations reduces the level of progesterone in the blood serum, thereby activating the generation of estrogen and nitric oxide (II). The latter limits the effect of stimulation to physiological limits, thereby preventing the effect of overexcitation. The positive correlation of nitric oxide (II) with estrogen suggests their relationship manifested by the effect on the physiological mechanisms of regulation of the reproductive cycle and the reproductive system as a whole.

In conclusion, it should be noted that further research is needed to find out whether dysfunction of the reproductive organs is associated with a decrease or increase in endogenous synthesis of the nitroxydergic system, since the regulation of induction of nitric oxide (II) may have important therapeutic value.

Like steroids, prostaglandins are a group of compounds that are somewhat similar in structure but can have very different effects. Prostaglandins are autocrine or paracrine regulators, not true hormones. These compounds are metabolized locally in many tissues, but if they are not degraded locally, they enter the circulatory system and are effectively neutralized by the degrading enzyme prostaglandin dehydrogenase in the lungs. Nitric oxide (II) and prostaglandins have a lot in common, and two molecules can be produced simultaneously in the same tissues. There is no doubt that nitric oxide (II) signaling is closely related to the prostaglandin pathway. The interaction between these two pathways occurs at several levels, due to the complexity of the redox chemistry of nitric oxide (II).

\section{References}

1. I.N. Bilalov, R.G. Karimov, Species and Sexual Specificity of Nitric Oxide Formation in the Body, XXII Congress of the Physiological Society named after I.P. Pavlov, Abstracts (Volgograd State Med. Univ. Publ. House, Volgograd, 2013), p. 63

2. R.G. Karimova, T.V. Garipov, Nitroxidergic system: influence of furoxan compounds, In: Veterinary medicine of domestic animals, Digest of articles (Kazan, 2009), pp. 85-88

3. A.G. Nezhdanov, M.N. Kochura, V.A. Safonov et al., The problem of diseases of the reproductive organs of dairy cows and the main ways of solving it, Proc. of the Int. sci.-pract. Conf. Actual problems of increasing the efficiency of the agro-industrial complex (Kursk, 2008), pp. 227-229

4. D. Nikulin, Synchronization of the sexual cycle of cows - "pros and cons", Niva Trans-Urals, 1(123), 80-81 (2015)

5. N.V. Pasko, Lipid peroxidation, antioxidant system and nitric oxide in postpartum disorders of uterine contractile function in cows (Cand. dissertation thesis) (Voronezh, 2009), $21 \mathrm{p}$.

6. S.G. Postovoy, The influence of prostaglandin F2 $\alpha$ preparations on the contractile function of the uterus of cows, Veter., 4, 36-38 (2007)

7. M.I. Retskiy, N.V. Ermakova, G.N. Bliznetsova et al., Dynamics of stable nitric oxide metabolites in cows with uterine subinvolution, Veter. Pathol., 2, 89-90 (2003)

8. V.A. Titova et al., Some aspects of intraovarian processes in cows, Collection of materials of the VIZh (Moscow, 2007), pp. 161-164

9. V.P. Khlopitsky, The use of biologically active drugs for the normalization of reproductive function in highly productive dairy cows, Praktik, 1, 43-47 (2009)

10. O.I. Sukmansky, V.P. Reutov, Gas transmitters: physiological role and participation in the pathogenesis of diseases, Adv. Physiol. Sci., 47, 30-58 (2016) 
11. O.S. Tarasova, S.I. Sofronova, D.K. Gainullina et al., Regulation of nitric oxide production by vascular endothelium during exercise: the role of thyroid hormones, Aviat. and envir. Med., 49(2), 55-62 (2015)

12. G. Basini, F. Grasselli, Nitric oxide in follicle development and oocyte competence, J. Reproduct., 150(1)

13. S.M. McCann, V. Rettori, The role of nitric oxide in reproduction, Proc. Soc. Exp. Biol. Med., 7-15 (1996)
14. K. Chwalisz, R.E. Garfield, Role of nitric oxide in implantation and menstruation, Hum. Reprod., 96-111 (2000)

15. O. Shynlova, R. Kwong, S. Lye, Mechanical stretch regulates hypertrophic phenotype of the myometrium during pregnancy, Reproduct., 70, 986-992 (2009)

16. F.J. Diaz et al., Regulation of progesterone and prostaglandin F2a production in the CL, Mol. Cell. Endocrinol. (2002) 